\title{
Advances in Atomic-resolution and Molecular-detection EELS
}

\author{
Ondrej L. Krivanek ${ }^{1,3}$, Niklas Dellby ${ }^{1}$, Tracy C. Lovejoy ${ }^{1}$, Ray F. Egerton ${ }^{2}$ and Peter Rez ${ }^{3}$ \\ 1. Nion R\&D, Kirkland, WA, USA \\ 2. Department of Physics, University of Alberta, Edmonton, Canada \\ 3. Department of Physics, Arizona State U., Tempe, AZ, USA
}

As MAS celebrates the $50^{\text {th }}$ anniversary of the Society's founding, the range and precision of available analytical techniques continue to grow rapidly. This contribution concentrates on electron energy loss spectroscopy (EELS), which has witnessed remarkable progress in the last 10 years.

Isaacson and Johnson predicted in 1975 that single atoms would eventually become detectable by EELS [1]. This prediction has now been fulfilled and surpassed. Four factors contributed:

a) efficient electronic detection of EELS data in parallel became standard from the 1980s on (e.g. [2]);

b) scanning transmission electron microscopes (STEMs) equipped with cold field emission guns (CFEGs) being able to provide $\mathrm{e}^{-}$probes $\sim 1 \AA$ in size at primary voltages $\leq 60 \mathrm{kV}$ (avoiding knockdamage in low $\mathrm{Z}$ materials), with currents $>100 \mathrm{pA}$ (e.g. [3]);

c) greatly improved STEM stability that allowed probing stationary single atoms with $>10^{10} \mathrm{e}^{\mathrm{e}}$.

d) advances in sample preparation, with monolayer materials available from around 2005 on.

Figure 1 shows remarkable EELS results, now 5 years old: background-subtracted $\mathrm{L}_{2,3}$ spectra of single $\mathrm{Si}$ atoms substituting for either two $\mathrm{C}$ atoms (4-fold substitutional site, top) or for a single $\mathrm{C}$ atom (3fold substitutional site, middle and bottom) in monolayer graphene. For the four-fold $\mathrm{Si}$, the experimental near-edge structure (ELNES) of the $\mathrm{L}_{2,3}$ edge closely corresponds to the theoretical prediction. For the three-fold $\mathrm{Si}$, the experiment-theory match is poor for in-plane substitutional $\mathrm{Si}$, but it improves greatly if the simulation allows the Si atom to "pop out" from the graphene plane [4]. These results demonstrate that EELS cutting edge capabilities have now progressed to ELNES analysis of the local atomic environment of single atoms. EDXS of single atoms has also become possible [5].

Similar progress has taken place in elemental and chemical mapping of crystals in zone axis orientation. Muller at al. demonstrated nearly ten years ago that an aberration-corrected CFEG STEM with an efficiently coupled EEL spectrometer can map elemental composition with atomic column resolution in less than 10 msec per pixel [6] while avoiding artefacts that arise when the collection efficiency is poor, and the technique has been improved since. The valence of elements such as Eu has also been mapped with atomic column resolution, and extension of atomic-resolution elemental mapping to three dimensions through tomography appears possible for radiation samples resistant to radiation damage.

Even more spectacular progress has taken place in EELS energy resolution. A ground potential monochromator coupled with ultra-high energy resolution EELS has improved the energy resolution to $7 \mathrm{meV}$, and progress to $5 \mathrm{meV}$ and further is expected [7]. The improved resolution has allowed vibrational spectroscopy to be carried out in the electron microscope [8]. Depending on what type of EELS signal is admitted into the EEL spectrometer, the analysis can be carried out either with nm-level spatial resolution [9], or in aloof geometry that minimizes radiation damage in sensitive samples [10]. The latter technique permits hydrogen analysis in many kinds of materials. An example of a vibrational spectrum that can now be obtained and used for molecular detection is shown in Fig. 2. 
A particularly promising future approach may become possible when a sample is scanned in a discontinuous manner, with the probe coming to rest in a few places separated by tens of $\mathrm{nm}$ of untouched sample [11]. In guanine, such "leapfrog scanning" produces essentially the same vibrational results as the aloof spectrum of Fig. 2. This indicates that analysing the types of organic compounds present in frozen hydrated biological sections at about $30 \mathrm{~nm}$ spatial resolution is likely to become feasible when a cryotransfer holder becomes available for our monochromated microscope system.

\section{References:}

[1] M Isaacson and D Johnson, Ultramicroscopy 1 (1975), p. 33.

[2] OL Krivanek, CC Ahn and RB Keeney, Ultramicroscopy 22 (1987), p. 103.

[3] OL Krivanek et al., Ultramicroscopy 123 (2012), p. 90.

[4] QM Ramasse et al., Nano Lett. 13 (2013), p. 4989; W Zhou et al., Phys. Rev. Lett. 109 (2012), p. 206803.

[5] TC Lovejoy et al., Appl. Phys. Lett. 100 (2012), 154101; K. Suenaga et al., Nat. Phot. 6, (2012), p. 545.

[6] DA Muller et al., Science 319 (2008), p. 1073.

[7] TC Lovejoy et al., these proceedings (2017).

[8] OL Krivanek et al., Nature 514 (2014), p. 209; Mizoguchi et al., Microscopy 63 (2014), p. 377.

[9] C Dwyer et al., Phys. Rev. Lett. 117 (2016), p. 256101.

[10] P. Rez et al., Nature Communications 7 (2016), p. 10945.

[11] RF Egerton et al., Microsc. Microanal. 22 (Suppl 3) (2016), p. 960.

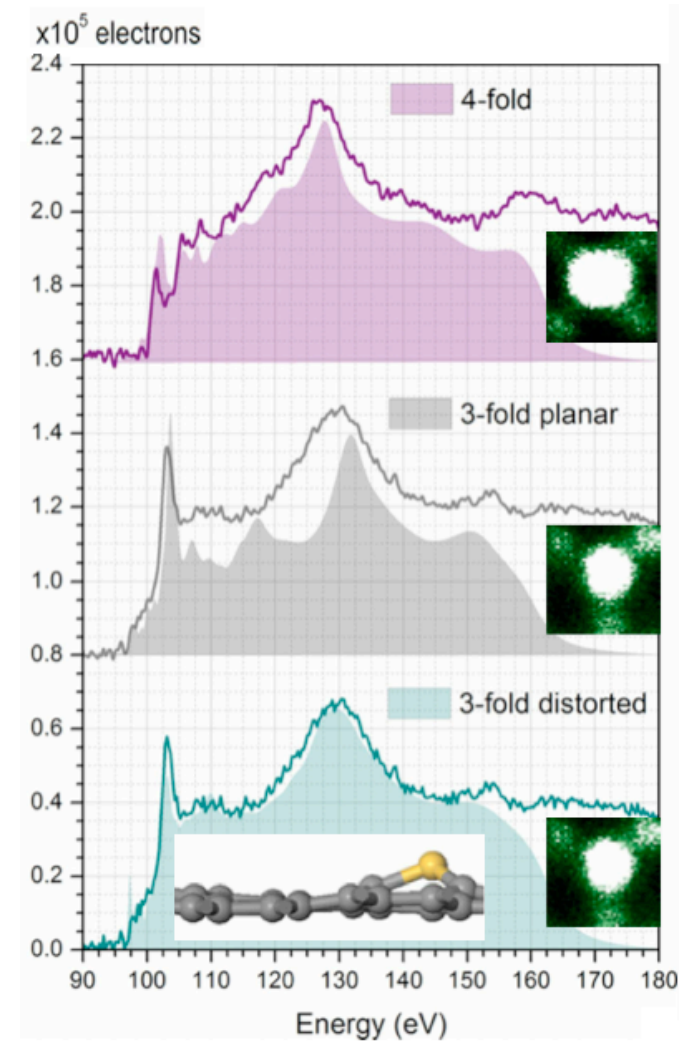

Figure 1. $\mathrm{Si} \mathrm{L}_{2,3}$ edges from single $\mathrm{Si}$ atoms embedded in graphene. Lines $=$ experiment, solid = theory. A good match for the 3-fold Si results when it's allowed to "pop-out" from the graphene plane (insert). See ref. [4].

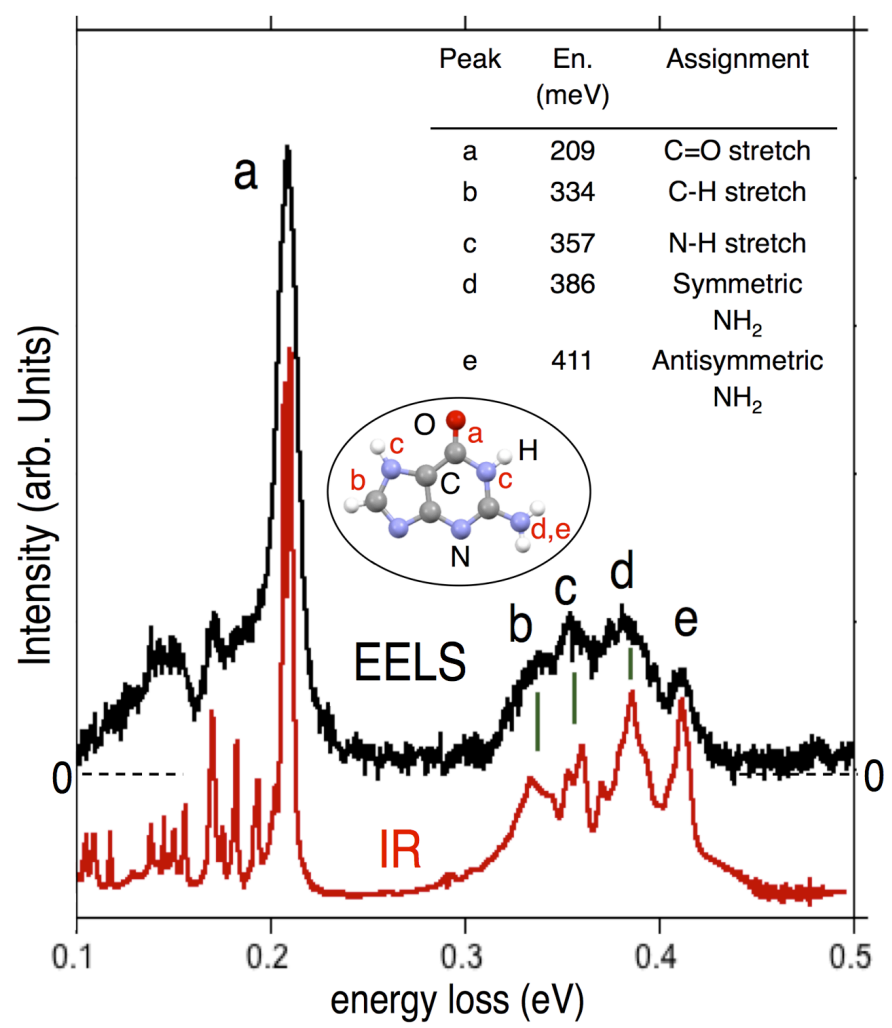

Figure 2. Vibrational EELS of guanine obtained in aloof geometry (probe-sample distance $=30$ $\mathrm{nm}$ ) compared to an infrared (IR) spectrum. The resolved peaks are labeled and related to structural features in the guanine molecule. See ref. [10]. 